\title{
Critical Networked Infrastructure Protection from Adversaries
}

\author{
Eduardo G. Quijano ${ }^{\mathrm{a}}$, David Ríos Insua ${ }^{\mathrm{b}}$, Javier Cano $^{\mathrm{a}, *}$ \\ ${ }^{a}$ Department of Computer Science and Statistics, Rey Juan Carlos University \\ ${ }^{b}$ Instituto de Ciencias Matemáticas, ICMAT-CSIC
}

\begin{abstract}
We use the adversarial risk analysis (ARA) framework to deal with the protection of a critical networked infrastructure from the attacks of intelligent adversaries. We deploy an ARA model for each relevant element (node, link, hotspot in link) in the network, using a Sequential Defend-Attack-Defend template as a reference. Such ARA models are related by resource constraints and result aggregation over various sites, for both the Defender and the Attacker. As a case study, we consider the protection of a section of the Spanish railway network from a potential terrorist attack.
\end{abstract}

Keywords: adversarial risk analysis, sequential defend-attack-defend model, critical infrastructure protection, network, railway transport

\section{Introduction}

As suggested in the Global Risks reports [1], security has become a major concern for governments and organisations worldwide. Large-scale terrorist attacks represent one of the most challenging current threats. Motivated by such events, an intense debate 5 has emerged in recent years about how to best protect critical infrastructures against terrorism.

The complexity of this global phenomenon reinforces the need to implement analytic tools to support decision makers, see e.g. [2] or [3] for recent accounts of various methodologies. 4] proposes several quantitative procedures to assess the vulnerability of critical

${ }^{*}$ Corresponding author. Camino del Molino s/n, Office B107, 28943 Fuenlabrada (Madrid), Spain. Tlf: +34914888411 . Fax +34914887500.

Email address: javier.cano@urjc.es (Javier Cano) 
infrastructures and establish optimal policies for mitigating such weaknesses. [5] apply game-theoretic based optimisation models to make critical infrastructures more resilient. From a more qualitative perspective, 6] discuss the usefulness of risk analysis as a valid tool for critical infrastructure protection. Other relevant works include [7] and [8], who provide overviews on strategies, models and research issues in terrorism risk analysis.

Most previous approaches to this problem have a game-theoretic flavour, see e.g. [9] and [10] for recent contributions. As such, they take account of the intelligent and adaptive nature of attackers. However, some of the assumptions on which they are based might not be realistic in the security context, mainly those in relation with common knowledge about the agents' preferences and probabilities, see [11]. Alternatively, we propose the use 20 of Adversarial Risk Analysis (ARA), see [12], to address the problem of how to best protect a critical infrastructure from intelligent terrorist threats. ARA provides one-sided prescriptive support to one of the intervening agents, based on a subjective expected utility model, treating the decisions of the adversaries as uncertainties.

We focus on problems in which an organisation needs to protect a critical networked 25 infrastructure (CNI) from terrorist threats. Examples of CNIs include water, oil or natural gas distribution systems, transportation routes, power grids, and telecommunication networks, see [4]. 13] provide a classification of systems defence and attack models. According to their terminology, we shall face cases of protecting a network from attacks by a single attacker with incomplete information. A recent review on security analysis of critical network systems may be seen in [14].

To address the protection of a network, we deploy an ARA model at each of its relevant elements, with possibly different types of resources and models for each type of element. Models will be related by resource constraints and aggregation of results over various targets for both the Defender and the Attacker. For illustrative purposes, we use an ARA 35 Sequential Defend-Attack-Defend template at all relevant elements, see [5], [15] or [16].

Our case study refers to deciding the optimal security resource allocation for a railway network whose operator fears the onslaught of a terrorist group. Railway systems constitute a preferred terrorist target: attacks against them entailing large numbers of casualties have taken place all over the world since the beginning of the 21st century, see 40 Table 1 for major examples. 
Table 1: Main terrorist attacks on railway: [17]

\begin{tabular}{ccc}
\hline Date & Country & Casualties \\
\hline $2001 / 8 / 1$ & Angola & $>250$ \\
$2004 / 3 / 11$ & Madrid, Spain & $>190$ \\
$2005 / 7 / 7$ & London, UK & $>50$ \\
$2006 / 7 / 11$ & Mumbai, India & $>180$ \\
$2007 / 2 / 17$ & Pakistan & $>60$ \\
\hline
\end{tabular}

Following these events, various studies on the vulnerability of railway transport have been conducted, e.g. [17]. They highlight that despite all security improvements, it is difficult to control and protect stations and tracks against terrorist attacks 24/7. 18] describe ongoing efforts in Europe to increase rail security.

\section{Methodology}

We present the ARA methodology proposed to deal with CNI protection from adversaries. A network operator (she, the Defender) needs to protect a CNI from terrorist threats (he, the Attacker). Networked infrastructures are composed of two types of elements: nodes and links. Sometimes links include a third type of element: hotspots of particularly high-value both for terrorists and the operator. For a railway network, nodes correspond to stations, whereas links refer to tracks connecting them. Hotspots could be, e.g., viaducts, tunnels, junctions or bridges along certain routes. To protect each element, we deploy an ARA model, see [16] for several templates. Different models could be used for different network element types. Such models will be related by resource constraints and aggregation of results over various sites for the Defender and the Attacker. Resources, as well as constraints, may vary for different types of elements.

As an illustration, consider a generic network element (node, link, hotspot), and assume we use a Sequential Defend-Attack-Defend model for it, see Figure 1 . Node $D_{1}$ corresponds to the Defender's initial decision $d_{1} \in \mathcal{D}_{1}$ about the deployment of countermeasures to deter and protect herself from the terrorist threat. After observing such defence, the Attacker decides his attack $a \in \mathcal{A}$ in node $A$. Node $S_{1}$ represents the out- 
come $s_{1} \in \mathcal{S}_{1}$ of such attack. Then, the Defender makes a decision $d_{2} \in \mathcal{D}_{2}$ at node $D_{2}$, possibly reshuffling resources to try to recover as best as she can from the attack. Node $S_{2}$ expresses the final result $s_{2} \in \mathcal{S}_{2}$ of the Defender's attempt to recover. Calligraphic ${ }_{65}$ letters denote the sets of possible decisions/outcomes. Dashed arrows into nodes $A$ and $D_{2}$ indicate the information respectively available to the Attacker and the Defender at the time they make the corresponding decisions. The Defender and the Attacker aggregate their consequences in nodes $c_{D}$ and $c_{A}$, respectively. The Defender's consequences depend on $\left(d_{1}, s_{1}, s_{2}\right)$, i.e., the effort to implement her initial protective measures, the initial 70 results of the attack and her recovery action. The results would be aggregated by the Defender over targets. Similarly, the consequences for the Attacker depend on $\left(a, s_{1}, s_{2}\right)$, i.e., the effort associated with his attack, its outcome, and the result after the Defender's subsequent move. The Attacker also aggregates results over targets. The agents then evaluate the results with their respective utilities $u_{D}$ and $u_{A}$.

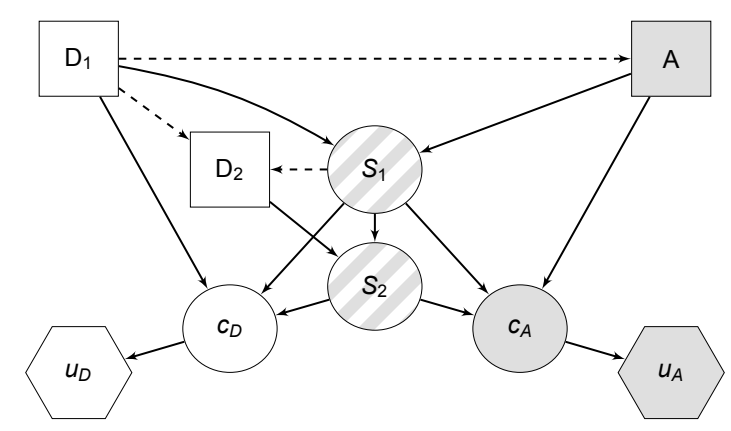

Figure 1: Influence diagram for the railway network case study.

The Defender aims at finding her optimal defence strategy $\left(d_{1}^{*}, d_{2}^{*}\left(d_{1}^{*}, s_{1}\right)\right)$, i.e. her optimal initial preventive defence $d_{1}^{*}$ and her optimal recovery defence $d_{2}^{*}\left(d_{1}^{*}, s_{1}\right)$, given $d_{1}^{*}$ and the attack result $s_{1}$. For this, she needs to assess her utility $u_{D}\left(c_{D}\right)$ and the probability models $p_{D}\left(s_{1} \mid d_{1}, a\right), p_{D}\left(s_{2} \mid s_{1}, d_{2}\right)$ and $p_{D}\left(a \mid d_{1}\right)$, respectively reflecting her beliefs about: (i) The initial attack outcome $s_{1}$ when defence $d_{1}$ has been deployed and attack $a$ is launched; (ii) The final outcome $s_{2}$ when the initial result of the attack is $s_{1}$ and the recovery action $d_{2}$ is taken; and (iii) What attack $a$ will the Attacker choose once he has observed the deployed defence $d_{1}$. With such assessments, we would solve 
the Defender's problem in Figure 2, based on the standard influence diagram reduction algorithm, see [19], proceeding through steps D0 D6.

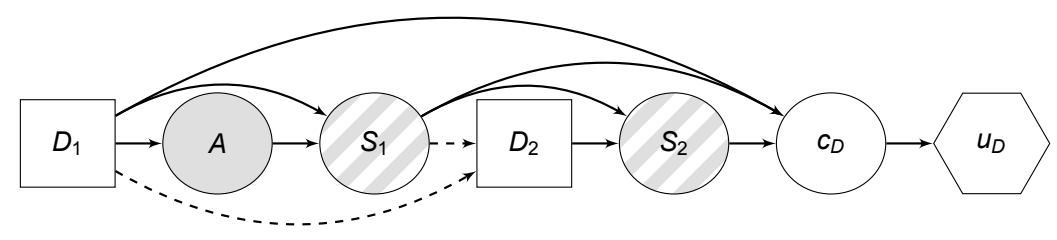

Figure 2: Defender's influence diagram.

D0. Aggregate results over targets.

D1. At node $c_{D}$, obtain the expected utilities

$$
\left(d_{1}, s_{1}, s_{2}\right) \rightarrow \psi_{D}\left(d_{1}, s_{1}, s_{2}\right)=\int u_{D}\left(c_{D}\right) p_{D}\left(c_{D} \mid d_{1}, s_{1}, s_{2}\right) \mathrm{d} c_{D}
$$

D2. At node $S_{2}$, get the expected utilities

$$
\left(d_{1}, s_{1}, d_{2}\right) \rightarrow \psi_{D}\left(d_{1}, s_{1}, d_{2}\right)=\int \psi_{D}\left(d_{1}, s_{1}, s_{2}\right) p_{D}\left(s_{2} \mid s_{1}, d_{2}\right) \mathrm{d} s_{2} .
$$

D3. At node $D_{2}$, compute and store the optimal values (and decisions)

$$
\left(d_{1}, s_{1}\right) \rightarrow\left\{\begin{array}{l}
d_{2}^{*}\left(d_{1}, s_{1}\right)=\arg \max _{d_{2} \in \mathcal{D}_{2}} \psi_{D}\left(d_{1}, s_{1}, d_{2}\right) \\
\psi_{D}\left(d_{1}, s_{1}\right)=\max _{d_{2} \in \mathcal{D}_{2}} \psi_{D}\left(d_{1}, s_{1}, d_{2}\right)
\end{array}\right.
$$

D4. At node $S_{1}$, obtain the expected utilities

$$
\left(d_{1}, a\right) \rightarrow \psi_{D}\left(d_{1}, a\right)=\int \psi_{D}\left(d_{1}, s_{1}\right) p_{D}\left(s_{1} \mid d_{1}, a\right) \mathrm{d} s_{1} .
$$

D5. At node $A$, obtain the expected utilities

$$
d_{1} \rightarrow \psi_{D}\left(d_{1}\right)=\int \psi_{D}\left(d_{1}, a\right) p_{D}\left(a \mid d_{1}\right) \mathrm{d} a
$$

D6. Finally, at node $D_{1}$, compute the optimal decisions

$$
d_{1}^{*}=\underset{d_{1} \in \mathcal{D}_{1}}{\arg \max } \psi_{D}\left(d_{1}\right)
$$


Provided that the number of portfolios is not too large, this can be accomplished by enumerating and evaluating all of them. Should that number be large, we could proceed by simulating the expected utility $\psi_{D}$ at a few $d_{1}$ and $d_{2}\left(d_{1}, s_{1}\right)$ values, fitting a regression metamodel $\widehat{\psi}_{D}\left(d_{1}, d_{2}\right)$, see e.g. [20], and optimising such metamodel.

The only nonstandard assessment in the above formulation is $p_{D}\left(a \mid d_{1}\right)$, which requires strategic thinking. To estimate it, the Defender may consider the Attacker's decision problem, see Figure 3. Assuming the Attacker is an expected utility maximiser, see [21], the Defender would need his probabilities $p_{A}\left(c_{A} \mid a, s_{1}, s_{2}\right), p_{A}\left(s_{2} \mid s_{1}, d_{2}\right), p_{A}\left(d_{2} \mid d_{1}\right.$, $\left.s_{1}\right)$ and $p_{A}\left(s_{1} \mid d_{1}, a\right)$ and utility $u_{A}\left(c_{A}\right)$ to obtain $a^{*}\left(d_{1}\right)$, his optimal attack given her defence $d_{1}$. However, the Defender typically lacks knowledge to assess them. Suppose we may model such uncertainty through random utilities and probabilities represented by $F=\left(U_{A}\left(c_{A}\right), P_{A}\left(c_{A} \mid \cdot\right), P_{A}\left(s_{2} \mid \cdot\right), P_{A}\left(d_{2} \mid \cdot\right), P_{A}\left(s_{1} \mid \cdot\right)\right)$. Then, for each $d_{1}$, she could proceed as follows

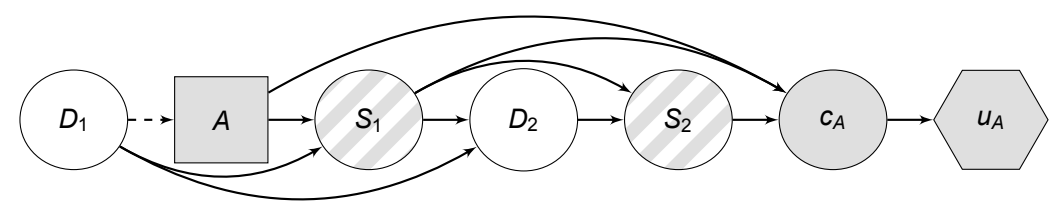

Figure 3: Attacker's influence diagram.

A0. Aggregate results over targets.

A1. At node $c_{A}$, obtain the (random) expected utilities

$$
\Psi_{A}\left(a, s_{1}, s_{2}\right)=\int U_{A}\left(c_{A}\right) P_{A}\left(c_{A} \mid a, s_{1}, s_{2}\right) \mathrm{d} c_{A} .
$$

A2. At node $S_{2}$, compute the (random) expected utilities

$$
\Psi_{A}\left(a, s_{1}, d_{2}\right)=\int \Psi_{A}\left(a, s_{1}, s_{2}\right) P_{A}\left(s_{2} \mid s_{1}, d_{2}\right) \mathrm{d} s_{2} .
$$

A3. At node $D_{2}$, get the (random) expected utilities

$$
\Psi_{A}\left(a, s_{1}\right)=\int \Psi_{A}\left(a, s_{1}, d_{2}\right) P_{A}\left(d_{2} \mid d_{1}, s_{1}\right) \mathrm{d} d_{2} .
$$


A4. At node $S_{1}$, obtain the (random) expected utilities

$$
\Psi_{A}\left(d_{1}, a\right)=\int \Psi_{A}\left(a, s_{1}\right) P_{A}\left(s_{1} \mid d_{1}, a\right) \mathrm{d} s_{1} .
$$

A5. At node $A$, obtain the (random) optimal decisions, given $d_{1}$,

$$
A^{*}\left(d_{1}\right)=\underset{a \in \mathcal{A}}{\arg \max } \Psi_{A}\left(d_{1}, a\right) .
$$

100 Then, a probability distribution of the optimal attack given $d_{1}$ would be $\int_{-\infty}^{a} p_{D}(\xi \mid$ $\left.d_{1}\right) \mathrm{d} \xi=\operatorname{Pr}\left(A^{*}\left(d_{1}\right) \leq a\right)$, which may be approximated through Monte Carlo simulation, sampling from $F$ and sequentially implementing steps A1 This provides us with the approximation $\hat{p}_{D}\left(a \mid d_{1}\right)$, the missing input to implement procedure D0 D6

We illustrate the application of the methodology with a case study which serves as template for other applications.

\section{Case Study Description}

\subsection{Motivation}

We consider the protection of the southwest section of the Spanish railway system against terrorist acts. Recent intelligence reports alert about the activation of a dormant cell established in Seville, integrated within the city for years without raising suspicion. This will be the Attacker. Note that several Al-Qaeda members have been arrested in Southern Spanish towns over the last years, see [22, 23, 24, 25, 26]. Terrorists intend to launch an attack in summer against the railway system and its users, due to the large population flows during the vacation period along the Andalusian coast.

The Defender in this case will be the railway operator, which is a public company attached to the Ministry of Public Works, responsible of security, in coordination with Homeland Security and Defence. It has a security budget to be distributed over various defensive resources across the network. The planning horizon is two months, corresponding to the summer period in which the terrorist alert would be active.

The section of the railway system under consideration is shown in Figure 4a, including as hotspots the Puente Genil's viaduct in the Córdoba-Málaga route, and the tunnels in Antequera and Jerez, in the Córdoba-Málaga and Seville-Cádiz routes, respectively. 
Trains circulating across the network are composed of three units, each of which consists of five coaches. A train maximum capacity is 780 seats, typically full in the summer.

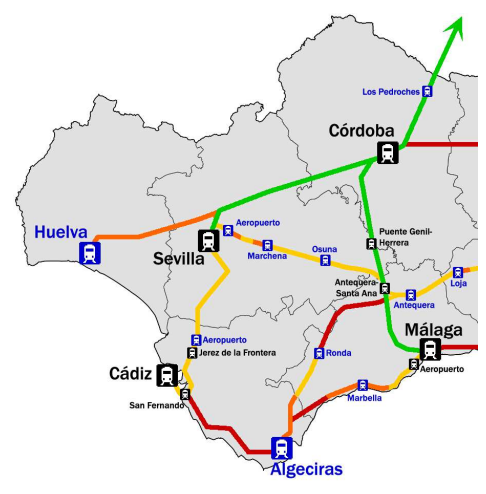

(a) Network map.

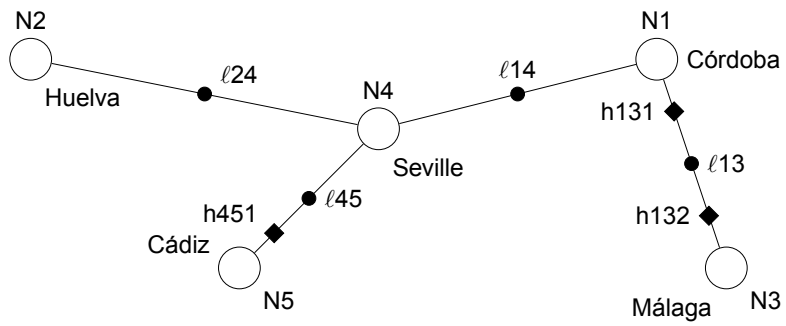

(b) Network scheme.

Figure 4: Railway network for case study.

\subsection{Defensive resources deployable by the railway operator}

Defensive resources can be fixed or mobile. The latter may be used to support recovery in the event of a successful attack, beyond the deterrent effect that all the resources, be they fixed or mobile, might have, as they are observed by the Attacker. By recovery we mean the detention of terrorists, not considering the protocols that the government and railway operator would carry out after an attack to: (i) Provide support to victims; and (ii) Restore service as soon as possible. These pertain to the domain of contingency plans, with a special unit attached to the Ministry of Defence trained to intervene in such emergencies. However, such unit is not responsible of capturing terrorists, being this the duty of Homeland Security. Thus, we will not include its eventual deployment in our model.

The defensive resources that the Defender considers are:

- Walk-through detectors. Guarantee all passengers are checked. Placed at entrances to platform areas. Aimed at detecting metal components of bombs. May produce queues.

- Cameras. Part of CCTV monitoring systems at stations, trains and tracks. 
- Additional illumination. Long-range self-powered directional spotlights, improving visibility at night.

- Fences. Prevent persons from accessing tracks at sensitive areas.

- Security staff. Patrol along stations, checking anyone suspicious. Periodically, will inspect hotspots along routes accompanied by detection dogs.

- Detection dogs. Trained to detect explosives. Must be always accompanied by security personnel.

- Helicopters. Each one is assigned to two crews. Randomly patrol along the network, communicating anomalies to mobile units at stations or hotspots.

Table 2 summarises features of the measures, including their associated unit costs over the two-month period. For security staff, we provide their unit gross salaries over such period. They will be outsourced to a private security company. Similarly, we have provided the bimonthly cost of hiring a detection dog. The costs entailed by helicopter patrols comprise crew salaries, equipment operation and maintenance. For fixed material taking into account typical lifetimes.

Table 2: Features of security measures and associated costs for the planning period

\begin{tabular}{ccccccc}
\hline & Measure & Station & Track & Hotspot & Type & Unit cost $(€)$ \\
\hline 1 & Metal detector & Yes & No & No & Fixed & 6,500 \\
2 & CCTV camera & Yes & No & Yes & Fixed & 650 \\
3 & Lamp post & No & Yes & Yes & Fixed & 3,000 \\
4 & Fence unit & No & Yes & Yes & Fixed & 4,200 \\
5 & Security guard & Yes & No & Yes & Mobile & 5,200 \\
6 & Detection dog & Yes & No & Yes & Mobile & 1,600 \\
7 & Helicopter & No & Yes & Yes & Mobile & 90,000 \\
\hline
\end{tabular}

\subsection{Terrorist strategies}

Consider now the motivations of the incumbent terrorist group, see [27]. They are usually assumed to be risk prone, their main aims being: (i) Inflict the largest number of 

civilians. In our case, note that:

- The places where the largest number of casualties can be caused are stations, due to the higher concentrations of people.

- The most devastating attack would be the detonation of an explosive inside a train as it enters a station. This would produce casualties within the train and the station platform, also damaging the train, the station and other assets.

- An attack using weapons of mass destruction (WMD) of chemical or biological nature would probably cause the greatest panic among civilians, recall the 1995 sarin gas release in Tokyo subway, see [17].

It is assumed that the terrorist cell has resources to launch a single attack over a single target over the incumbent period. We further assume that the attacks considered by the terrorists are: $\left(a_{1}\right)$ Place bomb in station; $\left(a_{2}\right)$ Place bomb inside a train and detonate when approaching a station; $\left(a_{3}\right)$ Place bomb along a route, and explode it as a train passes by; $\left(a_{4}\right)$ Place WMD inside a train and detonate it when approaching a station.

175 Note that for $a_{3}$, terrorists would ideally aim at putting the bomb as close as possible to a hotspot, so as to cause the largest damage. However, hotspots tend to be particularly well protected, thus becoming more perilous targets.

Lastly, in deciding their attack, the terrorists would take into account the following inherent threats: (i) Manipulation and transportation of weapons used in the attack, since, for instance, WMDs require more caution and care than explosives; (ii) The possibility of being detected by security forces before placing the explosive; and (iii) Introducing the explosive within the train and not being able to get off before detonation.

\section{Structuring the Case Study}

We specify the model elements from Section 2 in our case study. We perform it 185 stage-wise so as to serve as template for other CNI protection problems. 


\subsection{Network layout}

We start by identifying the network layout. A schematic representation of the network is in Figure 4b, labelling the stations $(\mathrm{N})$, routes $(\ell)$ and hotspots $(\mathrm{h})$ : h131 represents Puente Genil's viaduct; h132 and h451 stand for the tunnels in Antequera and Jerez, respectively.

Table 3 displays the main features of the stations (city population), routes (length) and hotspots (distance from depart) within the incumbent network. We use $g_{1}, \ldots, g_{12}$ for the twelve potential targets and $\mathcal{N}=\{1, \ldots, 5\}, \mathcal{L}=\{6, \ldots, 9\}$ and $\mathcal{H}=\{10,11,12\}$ for the sets of indices for nodes, links and hotspots, respectively, with $\mathcal{G}=\mathcal{N} \cup \mathcal{L} \cup \mathcal{H}$.

Table 3: Targets and main features

\begin{tabular}{|c|c|c|c|c|c|c|c|c|c|c|c|}
\hline \multicolumn{5}{|c|}{ Node $\equiv$ Station (city population) } & \multicolumn{4}{|c|}{ Link $\equiv$ Route $(\mathrm{km})$} & \multicolumn{3}{|c|}{ Hotspots (distance from depart) } \\
\hline$g_{1}$ & $g_{2}$ & $g_{3}$ & $g_{4}$ & $g_{5}$ & $g_{6}$ & $g_{7}$ & $g_{8}$ & $g_{9}$ & $g_{10}$ & $g_{11}$ & $g_{12}$ \\
\hline Córdoba & Huelva & Málaga & Seville & Cádiz & $\mathrm{Co}-\mathrm{Ma}$ & $\mathrm{Co}-\mathrm{Se}$ & $\mathrm{Hu}-\mathrm{Se}$ & $\mathrm{Se}-\mathrm{Ca}$ & h131 (v) & h132 (t) & $\mathrm{h} 451(\mathrm{t})$ \\
\hline 328.488 & 148.568 & 567.433 & 702.355 & 123.948 & 159 & 142 & 92 & 123 & Co-Ma (71) & Co-Ma (107.5) & $\mathrm{Se}-\mathrm{Ca}(84.8)$ \\
\hline
\end{tabular}

\subsection{First stage defences}

At each target, $D_{1}$ represents the countermeasures deployed by the Defender. We need to identify them, as well as the corresponding constraints. In our case, the first six resources in Table 2 may be deployed over specific targets, whereas the seventh one is a global resource deployed for the whole network. Let $x_{i, k}$ be the units of the $i$-th resource, $200 i=1, \ldots, 6$, deployed over target $g_{k}, k \in \mathcal{G}$. Thus, $x_{i, k}$ will be integer for $i=1, \ldots, 6$ and $k \in \mathcal{G}$. Let $\boldsymbol{x}_{i}=\left(x_{i, 1}, \ldots, x_{i, 12}\right)$, and $\bar{x}_{i}=\sum_{k=1}^{12} x_{i, k}$. Let $x_{7}$ be the number of helicopters deployed over the network. Unit costs $q_{i}, i=1, \ldots, 7$ are in Table 2 ,

The Defender imposes upper and lower bounds on the number of deployable units over each target, see Table 4 . For example, $1 \leq x_{1, k} \leq 2, k \in \mathcal{N}$ means that there must be mandatorily one but, at most, two metal detectors at each station (but none at links and hotspots).

Finally, let $B_{D}$ be the maximum budget available for new countermeasures, on top of the existing ones, for the relevant planning period. Then, the feasible first stage security 
Table 4: Bounds over deployable countermeasure units

\begin{tabular}{ccccccc}
\hline $1 \leq x_{1, k} \leq 2$ & $0 \leq x_{2, k} \leq 4$ & $0 \leq x_{3, k} \leq 2$ & $0 \leq x_{4, k} \leq 2$ & $3 \leq x_{5, k} \leq 4$ & $2 \leq x_{6, k} \leq 3$ & $1 \leq x_{7} \leq 2$ \\
$k \in \mathcal{N}$ & $k \in \mathcal{N} \cup \mathcal{H}$ & $k \in \mathcal{H}$ & $k \in \mathcal{H}$ & $k \in \mathcal{N} \cup \mathcal{H}$ & $k \in \mathcal{N} \cup \mathcal{H}$ & $k \in \mathcal{L} \cup \mathcal{H}$ \\
\hline
\end{tabular}

portfolios $d_{1}=\left(\boldsymbol{x}_{1}, \boldsymbol{x}_{2}, \boldsymbol{x}_{3}, \boldsymbol{x}_{4}, \boldsymbol{x}_{5}, \boldsymbol{x}_{6}, x_{7}\right)$ should also satisfy

$$
c_{\mathrm{inv}}=\sum_{i=1}^{6} q_{i} \bar{x}_{i}+q_{7} x_{7} \leq B_{D}
$$

In this case, the Defender's decision variables are integer. In other problems, they could be continuous or binary, or a mixture thereof.

\subsection{Attack strategies}

At each target, $A$ stands for the attack chosen by the terrorists. Define the binary decision variables $a_{j k} \in\{0,1\}, j=1, \ldots, 4, k \in \mathcal{G}$, with $a_{j k}=1$ meaning that terrorists decide to use attack $a_{j}$ against target $g_{k}$, and $a_{j k}=0$, otherwise. Since terrorists will launch one attack at most, we have

$$
\sum_{j=1}^{4} \sum_{k=1}^{12} a_{j k} \leq 1
$$

210 Additional logistic constraints apply to the Attacker's decision: (i) Attacks $a_{1}, a_{2}$ and $a_{4}$ can be carried out only close to stations. Thus, $a_{1 k}=a_{2 k}=a_{4 k}=0, k \in \mathcal{L} \cup \mathcal{H}$. (ii) Similarly, attack $a_{3}$ can be implemented only when the train is en route. Therefore, $a_{3 k}=0$, $k \in \mathcal{N}$.

In our case, the Attacker's decision variables are binary. In other problems they could be continuous, e.g. when describing attack effort, or integer, e.g. when describing number of attacking units deployed.

\subsection{Attack outcomes}

We reflect now the outcome of an eventual attack against a certain target. We assume binary outcomes taking values in $\mathcal{S}_{1}=\{0,1\}$. We define variables $s_{j k}^{(1)} \in \mathcal{S}_{1}, j=1, \ldots, 4$, 220 $k \in \mathcal{G}$, with $s_{j k}^{(1)}=1$ meaning a successful attack of type $a_{j}$ against target $g_{k}$, that is, the bomb explodes, and $s_{j k}^{(1)}=0$, otherwise. 
In other problems, the $s_{j k}^{(1)}$ variables could be continuous, reflecting, e.g., degree of destruction.

\subsection{Recovery decisions}

As $D_{2}$ is regarded, the Defender would use the available resources to try to capture the terrorists in case of a successful attack against a station or a hotspot. After an en route successful attack, she will shift such resources from the nearest location, whether a station or a hotspot. The decision variable $D_{2}$ takes values in $\mathcal{D}_{2}=\{0,1\}$, with $d_{2}=1$ meaning that recovery measures are mobilised, and $d_{2}=0$, otherwise.

In other problems, such decision variables could be integer or continuous.

\subsection{Recovery outcomes}

$S_{2}$ represents the result of the Defender's recovery action. It takes values in the set $\mathcal{S}_{2}=\{0,1\}$. We define binary variables $s_{j k}^{(2)} \in \mathcal{S}_{2}, j=1, \ldots, 4, k \in \mathcal{G}$, with $s_{j k}^{(2)}=1$ meaning that all terrorists - those still operational after a successful attack of type $a_{j}$ against target $g_{k}$-will be captured, and $s_{j k}^{(2)}=0$, otherwise. Again, in other problems, such state variables could be integer or continuous.

\subsection{Defender's consequences}

The impact of a successful attack may be tragic and devastating, see Table 1. The consequences considered relevant by the Defender in this case are: (i) Casualties, with associated cost $c_{\text {life }}$. (ii) Fear in population and negative perception of country's image, which we encompass in a variable $c_{\text {image }}$. (iii) Damage in fixed assets (tracks, stations,...). Their costs will be aggregated in a variable $c_{\text {fixed }}$. (iv) Damage in mobile elements, denoted by $c_{\text {train }}$. Table 5 summarises the relevant consequences. In case of no attack, there are no consequences for the operator, other than the incurred security investment.

Other examples of typical objectives of interest to Defenders in counterterrorism may be seen in [28].

The Defender's cost function will be described through

$$
c_{D}=c_{\text {inv }}+c_{\text {life }}+c_{\text {image }}+c_{\text {fixed }}+c_{\text {train }},
$$

aggregating consequences over all targets, effectively monetising them. 
Table 5: Attacks and potential consequences

\begin{tabular}{cccccc}
\hline Attack & Casualties & Image & Infrastructure & Station & Train \\
\hline$a_{1}$ & Yes & Yes & Yes & Yes & - \\
$a_{2}$ & Yes & Yes & Yes & Yes & Yes \\
$a_{3}$ & Yes & Yes & Yes & - & Yes \\
$a_{4}$ & Yes & Yes & - & - & - \\
\hline
\end{tabular}

\subsection{Attacker's consequences}

The Defender considers that the relevant multiple consequences for the incumbent terrorists are: (i) Preparation costs, $c_{\text {prep }}$. (ii) Number of terrorists killed or detained, for which the Attacker puts a value $c_{\text {life }}^{\prime}$. (iii) Whether they are able to wreak havoc among the population, since they also focus on image effects, $c_{\text {image. }}^{\prime}$ We aggregate the attributes into the Attacker's value function

$$
c_{A}=-c_{\text {prep }}-c_{\text {life }}^{\prime}+c_{\text {image }}^{\prime} .
$$

For other potential consequences of interest to terrorists, see [27].

\section{Assessing the Case Study}

Once we have structured the problem, we proceed with the required probability and utility assessments. We provide first those of the Defender and, then, the random probabilities and utilities of the Attacker required to forecast his attacks.

\subsection{Defender's assessments}

We assess the relevant probabilities, consequences and utilities in the Defender's problem. The available security budget for two months is $B_{D}=350,000 €$.

\subsubsection{Defender's probabilities}

Attack success probabilities. We start with the probabilities at $S_{1}$ nodes. For $j=1, \ldots, 4$, $k \in \mathcal{G}$, let us call $p_{j k}^{(1)} \equiv p_{D}\left(s_{j k}^{(1)}=1 \mid d_{1}, a_{j k}=1\right)$ the probability that a type $j$ attack 
against target $g_{k}$ is successful, conditional on the deployed countermeasures. The Defender believes that these probabilities depend on the factors introduced at the end of Section 3.3. We use

$$
p_{j k}^{(1)}=\left(1-\phi_{1}^{j k}\right) \cdot\left(1-\phi_{2}^{j k}\right),
$$

where $\phi_{1}^{j k}$ is the chance that the bomb explodes during manipulation or transportation, and $\phi_{2}^{j k}$ represents the probability that the terrorists are detected, and forced to abort the mission, which will depend on the type and number of deployed countermeasures. We are, thus, expressing that an attack will be successful only if the terrorists are not caught and the bomb explodes when planned. This will happen with probability $p_{j k}^{(1)}$.

To simplify the notation, we omit the dependence of $\phi_{1}, \phi_{2}$ on $(j, k)$. For $\phi_{2}$, we use an exponential model

$$
\phi_{2}\left(d_{1}\right)=1-\phi_{d} \cdot \exp \left(-\sum_{i=1}^{6} \gamma_{i} x_{i, k}-\gamma_{7} x_{7}\right),
$$

where $\gamma_{i}, i=1, \ldots, 7$ may be seen as deterrence parameters accounting for the fact that each additional unit of countermeasure is expected to decrease the chances of a successful attack, although in a decreasingly influential manner. Note that $1-\phi_{d}$ represents the detection probability if no additional measures are deployed.

Table 6 shows the values of $\phi_{1}, \phi_{d}$ and the $\gamma$ 's, assessed with the aid of operator experts. Observe that $\phi_{1}$ is significantly lower for WMDs $\left(a_{4}\right)$. Furthermore, terrorists have less chances of being detected when choosing attack $a_{3}$, explaining the greater value of $\phi_{d}$. Parameters $\gamma_{3}, \gamma_{4}$ (resp. $\gamma_{5}, \gamma_{6}$ ) apply only to attacks against tracks (resp. hotspots).

Table 6: Relevant parameters for the probability of successful attack

\begin{tabular}{cccccccccc}
\hline & $\phi_{1}$ & $\phi_{d}$ & $\gamma_{1}$ & $\gamma_{2}$ & $\gamma_{3}$ & $\gamma_{4}$ & $\gamma_{5}$ & $\gamma_{6}$ & $\gamma_{7}$ \\
\hline$a_{1}$ & 0.3 & 0.45 & 0.28 & 0.1 & - & - & 0.6 & 0.6 & - \\
$a_{2}$ & 0.4 & 0.35 & 0.28 & 0.13 & - & - & 0.69 & 0.69 & - \\
$a_{3}$ & 0.35 & 0.7 & - & - & 0.05 & 0.02 & 0.6 & 0.6 & 1.54 \\
$a_{4}$ & 0.1 & 0.4 & 0.2 & 0.13 & - & - & 0.69 & 0.69 & - \\
\hline
\end{tabular}

Note that we have used the same parameters for all locations. A more general approach could take into account site specificities reflecting vulnerabilities, at the expense of a bigger 
elicitation effort.

Recovery success probabilities. We assess now the probabilities at the $S_{2}$ nodes. We focus on $p_{j k}^{(2)} \equiv p_{D}\left(s_{j k}^{(2)}=1 \mid s_{j k}^{(1)}=1, d_{2}=1\right), j=1, \ldots, 4, k \in \mathcal{G}$, the probability of a successful recovery, when the Defender carries it out as a response to an accomplished attack. The Defender believes that $p_{j k}^{(2)}$ depends on the number and proximity of the nearest available mobile resources. We use an exponential model

$$
p_{j k}^{(2)}=1-p_{0 j} \cdot \exp \left(-\mu_{5} \tilde{x}_{5 k}-\mu_{6} \tilde{x}_{6 k}\right),
$$

to express the fact that the presence of security staff and detection dogs would increase the probability of capturing terrorists. Here, $1-p_{0 j}$ is the probability of a successful recovery after attack $a_{j}$, if no additional countermeasures are deployed. $\tilde{x}_{5 k}$ and $\tilde{x}_{6 k}$ stand for the number of security members and detection dogs shifted towards target $g_{k}$ from the nearest locations, respectively. Stations and hotspots are already protected, see Table 4, so there is no need to transfer new resources to them. Therefore, $\tilde{x}_{5, k}=x_{5, k}$ and $\tilde{x}_{6, k}=x_{6, k}$, when $k \in \mathcal{N} \cup \mathcal{H}$.

For an en route attack, mobile resources will be transferred from the nearest points (either a station or a hotspot). Parameters $\mu_{5}$ and $\mu_{6}$ model the efficiency of such countermeasures, attenuated by a remoteness factor $\lambda$ : intervention time is crucial and influenced by the distance $y$ between the attack location and the position from which resources need to be mobilised. We use the expressions

$$
\mu_{i}=\mu_{i 0} \cdot \exp \left(-\lambda_{i} \cdot y\right), i=5,6
$$

Let $\overline{a b}$ be the incumbent railway section, of length $y_{a b}$; let $\left(x_{5 a}, x_{6 a}\right)$ and $\left(x_{5 b}, x_{6 b}\right)$ be the number of deployed mobile resources at extremes $(a, b)$ of such section. If the chosen route contains no hotspots - as e.g. the Córdoba-Seville route in Figure $4 \mathrm{~b}$ - the Defender believes that the Attacker will place the bomb at a random point $\theta$ sufficiently far from protected places ( $a \equiv$ Córdoba and $b \equiv$ Seville stations in our example), which we model with a triangular distribution. Then, $\theta \mid d_{1} \sim \mathcal{T} \operatorname{ri}(a, b, m)$, where $m=y_{a b}\left(x_{5 a}+x_{6 a}+\right.$ 1)/ $\left(x_{5 a}+x_{6 a}+x_{5 b}+x_{6 b}+2\right)$ is the mode of the triangular distribution, reflecting that terrorists would tend to put the bomb as far as possible from the most protected place. 
The shortest distance $y=\min (\overline{\theta a}, \overline{\theta b})$ will determine from which station will the mobile resources be transferred, see Section 5.1.1 For a route containing one or more hotspotsas e.g. the Seville-Cádiz or Córdoba-Málaga routes in Figure $4 \mathrm{~b}$-we would proceed in a similar fashion, using a mixture of triangular distributions to account for the fact that hotspots are also protected with mobile resources.

Based on expert judgement, the Defender estimates the parameters $\mu_{50}=0.28, \mu_{60}=$ 0.13 and $\lambda_{5}=\lambda_{6}=0.03$. She also estimates $p_{0}=0.8$ for $a_{3}$, and $p_{0}=0.4$ for the other attack types. As before, it could be the case that these parameters are target dependent to reflect, e.g., accessibility, if the Defender wishes to model so.

\subsubsection{Defender's consequences forecasts}

We provide forecasting models for the relevant consequences in Section 4.7

Forecasting human losses. For attacks $a_{1}$ and $a_{2}$, we define a binomial random variable $z_{s}$ to forecast the number of casualties that would occur at the target station,

$$
z_{s} \mid g_{k} \sim \mathcal{B} \operatorname{in}\left(n_{s}, p_{s}\right), k \in \mathcal{N}
$$

$n_{s}$ depends on the station and represents the maximum number of people potentially affected by the blast. Based on expert judgement, we assess that $n_{s}$ is about $0.5 \%$ of the corresponding population size at each station, see Table 3. $p_{s}$ is the probability of a person being killed if present when the attack occurs. In this case, the Defender assumes the same probability for all stations, although she has uncertainty about its value, modelled through a beta distribution $p_{s} \sim \mathcal{B} e\left(\alpha_{s}, \beta_{s}\right)$. To assess the expected probability, $E\left[p_{s}\right]$, we rely on previous similar terrorist attacks, see [17]. First, we estimate the expected number of casualties at 50 for $g_{5}$, i.e. $E\left[z_{s} \mid g_{5}\right]=50$, from which we obtain $E\left[p_{s}\right]=0.081$. The Defender also assessed a value for $\operatorname{Var}\left[p_{s}\right]=0.001$. These two determine, in turn, the values of $\alpha_{s}=5.90$ and $\beta_{s}=67.27$. Finally, we obtained the expected number of casualties for the other stations, see Table 7 using their population size ratios.

We consider now the casualties that would occur within the train, either when entering the station $\left(z_{e}\right)$, or en route $\left(z_{r}\right)$. For attack $a_{2}$, we assume that only passengers in the coach where the bomb is placed would be affected. Under attack $a_{3}$, any passenger 
Table 7: Expected number of victims after a bomb attack

\begin{tabular}{cccccccc}
\hline Target & $g_{1}$ & $g_{2}$ & $g_{3}$ & $g_{4}$ & $g_{5}$ & Train in station & En route train \\
\hline Casualties & 133 & 60 & 229 & 283 & 50 & 49 & 156 \\
\hline
\end{tabular}

travelling within the unit (five coaches) where the bomb has been placed could be potentially affected. We use binomial distributions $z_{e} \sim \mathcal{B} i n\left(n_{e}, p_{e}\right)$ and $z_{r} \sim \mathcal{B} i n\left(n_{r}, p_{r}\right)$, being $n_{e}=52$ and $n_{r}=260$ the maximum capacity of a coach and a unit, respectively. The inherent uncertainty over the probabilities is reflected through beta distributions $p_{e} \sim \mathcal{B} e\left(\alpha_{e}, \beta_{e}\right)$ and $p_{r} \sim \mathcal{B} e\left(\alpha_{r}, \beta_{r}\right)$, with expected values $E\left[p_{e}\right]=0.95$ and $E\left[p_{r}\right]=0.6$, and moderate variances 0.02 and 0.01 , assessed by operator experts.

In an attack with WMDs $\left(a_{4}\right)$, approximately half of the passengers onboard are expected to be contaminated. We use a binomial distribution $z_{w} \sim \mathcal{B}$ in $\left(n_{w}, p_{w}\right)$ for the number of victims, with $n_{w}=780$ the maximum train capacity, and a beta distribution for the incumbent probability $p_{w} \sim \mathcal{B} e\left(\alpha_{w}, \beta_{w}\right)$, with $E\left[p_{w}\right]=0.5$ and $\operatorname{Var}\left[p_{w}\right]=0.008$. In addition, all people present at the station at the time of attack within a certain distance from the WMD focus would be affected. For simplicity, we consider the range of the WMD similar to that of a conventional explosion. Subsuming both contributions, we obtain the estimations in Table 8

Table 8: Expected number of victims after a WMD attack

\begin{tabular}{cccccc}
\hline Target & $g_{1}$ & $g_{2}$ & $g_{3}$ & $g_{4}$ & $g_{5}$ \\
\hline Casualties & 523 & 450 & 619 & 673 & 440 \\
\hline
\end{tabular}

The associated expected costs are obtained multiplying the expected casualty count by $q_{\mathrm{svl}}$, the statistical value of life for the Defender, see [29]. We use $q_{\mathrm{svl}}=2.04 \mathrm{M} €$. We then aggregate the costs due to casualties through

$$
c_{\text {life }}=q_{\mathrm{svl}}\left[\sum_{k=1}^{5} a_{1 k} z_{s}+\sum_{k=1}^{5} a_{2 k}\left(z_{s}+z_{e}\right)+\sum_{k=6}^{12} a_{3 k} z_{r}+\sum_{k=1}^{5} a_{4 k}\left(z_{s}+z_{w}\right)\right] .
$$

$c_{\text {life }}$ inherits the uncertainty in $z_{s}, z_{e}, z_{r}$ and $z_{w}$, described above.

Forecasting image losses. These would depend on the attack chosen by the terrorists and the effectiveness of the Defender's recovery action. Should all terrorists be detained, the 
impact on the country's image would be lower. We use a truncated (at zero) normal model

$$
p_{D}\left(c_{\text {image }} \mid a, s_{1}=1, s_{2}\right) \sim \mathcal{T} \mathcal{N}\left(\mu_{a}, \sigma_{a}^{2}\right) .
$$

Table 9 shows the expected values and variances assessed by operator experts.

Table 9: Expected image costs and associated standard deviation (M€)

\begin{tabular}{ccccc}
\hline & $a_{1}$ & $a_{2}$ & $a_{3}$ & $a_{4}$ \\
\hline$s_{2}=0$ & $29(14)$ & $29(14)$ & $20(9)$ & $35(17)$ \\
$s_{2}=1$ & $15(7)$ & $15(7)$ & $10(6)$ & $18(8)$ \\
\hline
\end{tabular}

Forecasting material losses. For costs associated with infrastructure damage, we have assessed the construction value of different assets, assuming an affected area of $500 \mathrm{~m}$ around the blast. Denote the costs in tracks and signals, tunnels and viaducts by $q_{\mathrm{tas}}$, $q_{\text {tun }}$ and $q_{\text {via }}$, respectively. We have used as reference the cost estimation of Atocha station during the Madrid train bombings, see [30], displayed in Table 10.

Table 10: Associated costs (M€) with damages over infrastructures

\begin{tabular}{ccccc}
\hline & $q_{\text {tas }}$ & $q_{\text {tun }}$ & $q_{\text {via }}$ & $q_{\text {sta }}$ \\
\hline Cost & 1.7 & 4.62 & 2.00 & 0.81 \\
\hline
\end{tabular}

To discriminate whether a bomb has been put sufficiently close to a tunnel or a viaduct under attack $a_{3}$, we define a binary variable $\eta \in\{0,1\}$, with $\eta=1$ if the shock wave affects the hotspot (i.e. when $\theta<500 \mathrm{~m}$ ), and $\eta=0$, otherwise. Since material costs are relatively low compared with life losses, we do not take into account their uncertainty. Then, their overall value is $c_{\text {fixed }}=\left(q_{\mathrm{tas}}+q_{\mathrm{sta}}\right) \sum_{j=1}^{2} \sum_{k=1}^{5} a_{j k}+q_{\mathrm{tas}} \sum_{k=6}^{9} a_{3 k}+\left(q_{\mathrm{tas}}+\eta \cdot q_{\mathrm{via}}\right) a_{310}+\left(q_{\mathrm{tas}}+\eta \cdot q_{\mathrm{tun}}\right) \sum_{k=11}^{12} a_{3 k}$.

The first term corresponds to attacks $a_{1}$ and $a_{2}$. The remaining refer to $a_{3}$ attacks, distinguishing between whether the bomb is placed at a hotspot or not. Note that attack $a_{4}$ would not imply material losses to the Defender. 
We also estimate the costs associated with damages in the train, differentiating whether it is entering a station (just the coach carrying the bomb is affected) or en route (five coaches suffer the impact). Each unit costs $q_{\text {unit }}=5.77 \mathrm{M} €$. Then, the cost caused by damages in trains is

$$
c_{\text {train }}=q_{\text {unit }}\left(\frac{1}{5} \sum_{k=1}^{5} a_{2 k}+\sum_{k=6}^{12} a_{3 k}\right) .
$$

We disregard the uncertainty associated with material losses, as they are comparatively much smaller than human or image costs.

\subsubsection{Utility function $u_{D}$}

The Defender is constant risk averse with respect to the $\operatorname{cost} c_{D}$, see [31]. Thus, her utility function is strategically equivalent to $u_{D}\left(c_{D}\right)=-\exp \left(k_{D} \cdot c_{D}\right)$, with risk aversion coefficient $k_{D}>0$. With the aid of experts, we assessed that $k_{D}=1.4 \cdot 10^{-3}$, using the probability equivalent (PE) method, see [32].

\subsection{Defender's assessments of Attacker's probabilities and utilities}

We discuss now the Defender's assessment of quantities corresponding to the Attacker's problem. She requires these to forecast the attacks to be faced.

\subsubsection{Attacker's probabilities}

Attack success (random) probabilities. For the Attacker's beliefs over $S_{1}$ nodes, we model $P_{A}\left(s_{j k}^{(1)}=1 \mid d_{1}, a_{j k}=1\right)$, the random probability of a successful attack $a_{j}$ against target $g_{k}$, as a beta distribution, with mean $p_{j k}^{(1)}$, see (11), and variance $\delta_{1}$. The Defender has little uncertainty about such assessment, setting $\delta_{1}=\min \left(0.1,0.95 \cdot p_{j k}^{(1)} \cdot\left(1-p_{j k}^{(1)}\right)\right)$. This upper bound on $\delta_{1}$ guarantees that the beta coefficients are positive.

Recovery success (random) probabilities. Similarly, we assess the Defender's beliefs about the random probability that the terrorists give to a successful recovery, $P_{A}\left(s_{j k}^{(2)}=1 \mid s_{j k}^{(1)}=\right.$ $\left.1, d_{2}=1\right)$, as a beta distribution with mean $p_{j k}^{(2)}$, see (2), and moderate variance, estimated by the Defender at $\delta_{2}=\min \left(0.15,0.95 \cdot p_{j k}^{(2)} \cdot\left(1-p_{j k}^{(2)}\right)\right)$. 


\subsubsection{Attacker's consequences (random) forecasts}

We assess all relevant consequences for the Attacker described in Section 4.8. The costs $c_{\text {prep }}$ associated with the preparation of an attack would depend on the type of attack, aggregating expenses such as fabrication of the explosive, train tickets and transportation to the target location. However, we finally disregarded these costs since they were much lower than other relevant costs.

Forecasting human losses. We consider the number $v$ of terrorists captured or killed during the attack. The Defender uses a binomial distribution to model $v$

$$
v \mid d_{1} \sim \mathcal{B} \operatorname{in}\left(t, p_{t}\right),
$$

where $t$ is the number of terrorists and $p_{t}$ is the probability that a terrorist is killed or detained in that phase. The Defender believes that the terrorist cell will be composed of three, four or five members, respectively with probabilities $0.2,0.5$ and 0.3 . She also models $p_{t}$ as $1-\left(1-\phi_{1}^{j k}\right)\left(1-\phi_{2}^{j k}\right)$, with $\phi_{1}^{j k}$ and $\phi_{2}^{j k}$ as in (1), expressing that a terrorist will escape after the attack only if he is not detected and the bomb explodes when planned. This will happen with probability $1-p_{t}$. As for the terrorist life value, we use again the statistical value of life (adapted to the country in our scenario), evaluating the possibility of being killed or detained at 300,000 €, see [33] or [34]. Thus, the total life costs faced by the terrorists would be

$$
c_{\mathrm{life}}^{\prime}=q_{\mathrm{svl}}^{\prime} \cdot v \text {. }
$$

In case of successful recovery by the Defender, we assume that the remaining terrorists, $t-v$, will be killed or detained. Thus, the expected global life costs for the Attacker would be $c_{\text {life }}^{\prime}=q_{\mathrm{svl}}^{\prime} \cdot t$. The uncertainty in $v$ and $t$ would be propagated to $c_{\text {life }}^{\prime}$.

Forecasting image losses. The Defender estimates that terrorists will have similar expectations to hers as regards image costs. However, she believes that they will also put a value to the number of potential victims caused in the attack. Then, she uses a truncated normal distribution, whose expected value equals $\mu_{a}$ times a factor $\kappa$ accounting for the relative impact - in terms of the expected number of casualties - of each attack type. From Table 7 and taking $g_{5}$ as a reference, we obtain $\kappa=(2.66,1.20,4.58,5.66)$ 
for $g_{1}, g_{2}, g_{3}$ and $g_{4}$, respectively. The Defender also acknowledges a greater uncertainty, choosing a bigger variance. Thus,

$$
c_{\text {image }}^{\prime} \mid a, s_{1}=1, s_{2} \sim \mathcal{T N}\left(\kappa \cdot \mu_{a}, 10 \cdot \sigma_{a}^{2}\right) .
$$

\subsubsection{Attacker's (random) utility function}

We assume that terrorists are constant risk prone in benefits. Therefore, their utility function will be strategically equivalent to

$$
u_{A}\left(c_{A}\right)=\exp \left(k_{A} \cdot c_{A}\right), k_{A}>0 .
$$

Given the uncertainty about the risk proneness coefficient $K_{A}$, we consider the random utility model for the terrorists

$$
U_{A}\left(c_{A}\right)=\exp \left(k_{A} \cdot c_{A}\right), k_{A} \sim \mathcal{U}\left(0, K_{A}\right)
$$

Based on the PE method, we assessed the maximum risk proneness coefficient in the Attacker's utility function at $K_{A}=2.5 \cdot 10^{-3}$.

\section{Evaluating the Case Study}

\subsection{Approximating the expected utility}

In the case study, there are 36 decision variables and 81 constraints, yielding a number of feasible defence portfolios in the range of several hundreds of millions. A strategy based on evaluating each portfolio is computationally infeasible. Alternatively, as suggested in

375 Section 2, we have trained a neural network (NN) metamodel with one hidden layer to approximate the Defender's expected utility function and then maximised it, see [35]. Our choice for a NN model is based on its universal approximation features, see [36]. Note that the metamodel was fitted only for decision variable $d_{1}$ since, in our case, the optimal $d_{2}$ is easily obtained: if attack is successful, then recovery resources are mobilised; and they are not, otherwise.

Our NN architecture is shown in Figure 5, corresponding to a multilayer perceptron with one hidden layer, see [35]. Our input variables will be the decision variables, which for convenience, we have renamed as $\iota_{1}=x_{1,1}, \iota_{2}=x_{1,2}, \ldots, \iota_{35}=x_{6,12}, \iota_{36}=x_{7}$. As for 
the hidden layer, we tried different values for the number of nodes, finding appropriate fit for 10 nodes. The output of the $h$-th hidden node, using a logistic sigmoid activation function, is designated $v_{h}$. As usual in the NN methodology, bias nodes were used in both the hidden and input layers (setting their inputs to 1). The weight on the link connecting the $i$-th input node (bias node) to the $h$-th hidden node is denoted $w_{i, h}$ $\left(w_{b, h}\right), i=1, \ldots, 36, h=1, \ldots, 10$. Analogously, $w_{h}\left(w_{b}\right)$ stands for the weight from the $390 h$-th hidden node (bias node) to the output node, which is the expected utility $\psi_{D}\left(d_{1}\right)$ of the corresponding defence configuration $d_{1}=\left(x_{1,1}, x_{1,2}, \ldots, x_{6,12}, x_{7}\right)$, which we try to approximate with the expected response $\widehat{\psi}_{D}\left(d_{1}\right)$ of the NN model.

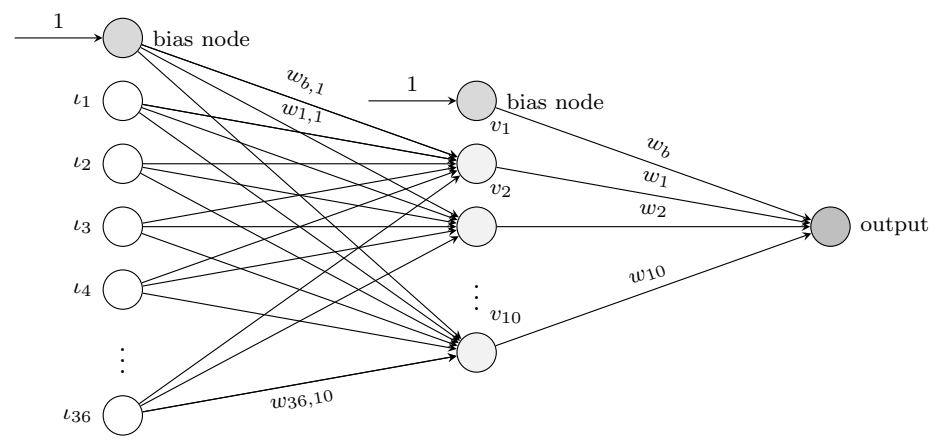

Figure 5: Neural network used to fit the Defender's expected utility function.

We used the Matlab built-in Bayesian regularization algorithm within the Neural Network Toolbox to estimate the NN, with 5,000 training points, randomly generated from the set of feasible defence portfolios. $80 \%$ were used for training and the remaining $20 \%$ for network generalisation. It was trained for 728 iterations. The best mean squared error (mse, Matlab default performance measure) was 0.0095. The fit between target and output values was reasonably good, with $R$ values above 0.85 for the training set considered.

\subsection{Optimal defence}

Once the Defender's expected utility function was approximated with $\widehat{\psi}_{D}\left(d_{1}\right)$, we optimised this to approximate, in turn, the Defender's best defensive portfolio $d_{1}^{*}$. To do so, we used a genetic algorithm, 37], with fitness function $\widehat{\psi}_{D}\left(d_{1}\right)$. We employed 
the MATLAB built-in ga function within the Global Optimization Toolbox with default options: the optimisation stopped when the weighted average relative change in the best fitness function value over StallGenLimit $=50$ generations was less than TolFun $=10^{-6}$.

The optimal defence configuration is shown in Table 11, with approximate expected utility $\widehat{\psi}_{D}\left(d_{1}^{*}\right)=-1.09$ and incurred costs of $346,450 €$, almost exhausting the available budget.

Table 11: Defender's optimal portfolio $d_{1}^{*}$ and associated costs $(€)$

\begin{tabular}{cccccccccccccc}
\hline & $g_{1}$ & $g_{2}$ & $g_{3}$ & $g_{4}$ & $g_{5}$ & $g_{6}$ & $g_{7}$ & $g_{8}$ & $g_{9}$ & $g_{10}$ & $g_{11}$ & $g_{12}$ & Cost \\
\hline$x_{1}$ & 2 & 1 & 2 & 2 & 1 & - & - & - & - & - & - & - & 52,000 \\
$x_{2}$ & 3 & 2 & 4 & 4 & 1 & - & - & - & - & 3 & 4 & 0 & 13,650 \\
$x_{3}$ & - & - & - & - & - & 0 & 0 & 0 & 0 & 2 & 2 & 2 & 18,000 \\
$x_{4}$ & - & - & - & - & - & 0 & 0 & 0 & 0 & 2 & 2 & 2 & 25,200 \\
$x_{5}$ & 3 & 2 & 3 & 3 & 2 & - & - & - & - & 2 & 3 & 3 & 109,200 \\
$x_{6}$ & 3 & 3 & 3 & 3 & 3 & - & - & - & - & 3 & 3 & 3 & 38,400 \\
$x_{7}$ & & & & & & & 1 & & & & & 90,000 \\
\hline Cost & 35,350 & 23,000 & 36,000 & 36,000 & 22,350 & 90,000 & 31,550 & 37,400 & 34,800 & $\mathbf{3 4 6 , 4 5 0}$ \\
\hline
\end{tabular}

${ }_{410}$ As we can observe, approximately $43 \%$ of the proposed investment would be on mobile resources (security guards, $x_{5}$, and dogs, $\left.x_{6}\right) ; 31 \%$ on fixed elements $\left(x_{1}-x_{4}\right)$; and the remaining $26 \%$ would be allocated to rent one helicopter $\left(x_{7}\right)$. Around $62 \%$ of the mobile resources $\left(x_{5}, x_{6}\right)$ and $67 \%$ of the CCTV cameras $\left(x_{2}\right)$ would be assigned to stations $\left(g_{1}-g_{5}\right)$; the rest to hotspots $\left(g_{10}-g_{12}\right)$. Tracks $\left(g_{6}-g_{9}\right)$ would be covered just by the helicopter. With regards to the stations, those located in bigger cities $\left(g_{4}, g_{3}\right)$ would be better protected by both material and human resources. Similarly, tunnels $\left(g_{11}, g_{12}\right)$ would be better defended than the viaduct $\left(g_{10}\right)$.

We should mention that many other portfolios attained close expected utilities. In most cases, such portfolios differed from the optimal one in a reduced number of variables, usually entailing just a redistribution of the same resources over different targets. Thus, the solution displayed in Table 11 should be taken as tentative. The Defender could further refine it through sensitivity analysis. 


\subsection{Analysing the Attacker's strategy}

Several remarkable features can be extracted from an analysis of the Attacker's problem. Our first interest lies in ascertaining which attack would the terrorists most likely undertake given that the Defender deploys the optimal portfolio $d_{1}^{*}$. To do so, we need to sample from the Attacker's random utility and probabilities conditional on $d_{1}^{*}$, performing steps $\mathrm{A} 0 \mathrm{A5}$ in Section 2 Figure 6 shows the expected probabilities of attack $a_{j}$ over target $g_{k}$ being optimal for the Attacker (solid line), with .95 probability bands around them plotted as dotted lines. We have also included the probability of no attack, denoted by $(0,0)$, although its expected value is negligible, therefore suggesting a clear and present danger of attack. Figure 7 displays plotbars with the expected probabilities of attack $a_{j k}$ being optimal, for each target in the network.

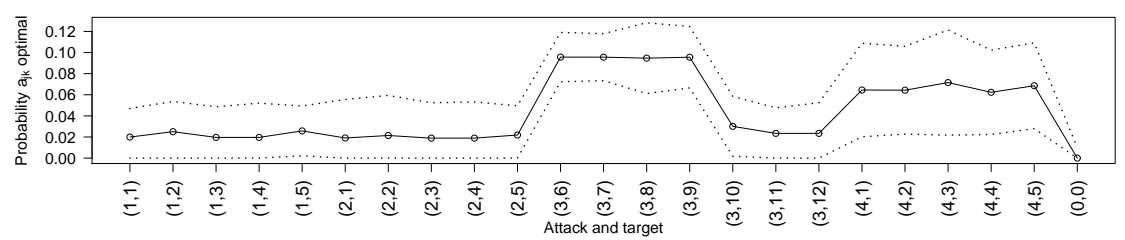

Figure 6: Probability of attack $(j, k)$ being optimal.

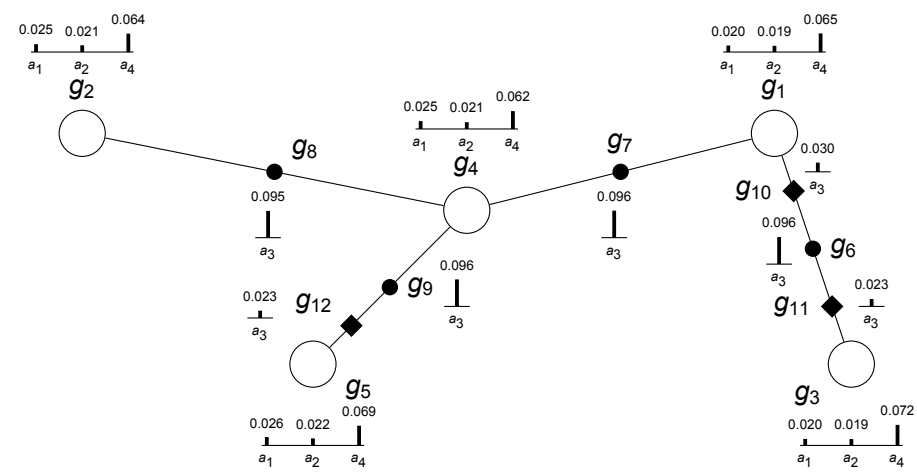

Figure 7: Probability of attack $a_{j}$ over target $g_{k}$ being optimal.

Thus, the most likely attack would be $a_{3}$ (bomb along a route) against any route, ${ }_{435} g_{6}, \ldots, g_{9}$ (the probabilities are similar in value). This suggests that although the potential consequences of attack $a_{3}$ are the least onerous for the Defender, the terrorists would have 
the greatest chance of being successful when choosing it. A similar reasoning explains why attack $a_{4}$ seems the second preference for the Attacker. Although its success probability is close to that of $a_{1}$ and $a_{2}$, the entailed consequences for the Defender would be more severe in terms of image costs. Other possibilities (attack $a_{3}$ against hotspots; and attacks $a_{1}$ and $a_{2}$ ) have equally low probabilities of being the optimal choice for terrorists. Note, though, that the probability distribution of the optimal attack is quite flat, with relatively uniform values for different attack-target combinations. This justifies the need to protect all targets, see Table 11

The Defender should be interested also in the outcome $s_{j k}^{(1)}$ for each possible attack and target. For attacks along routes, we had to sample the point $\theta$ at which terrorists would place the bomb. We obtained $\theta>500 \mathrm{~m}$ in almost all replications, meaning that the terrorists would put the bomb far from hotspots, which would be therefore unaffected by the blast. This seems plausible, since hotspots are well protected under the optimal defensive portfolio. Figure 8a shows the expected success probabilities (solid line), together with .95 probability bands (dotted lines). The attacks with highest probability of being successful are those perpetrated against routes (attack $a_{3}$; targets $g_{6}, g_{7}, g_{8}, g_{9}$ ), since they are the worst defended targets. The success probability in such cases is around 0.1 , about ten times greater than in the rest of attacks, although there is more uncertainty.

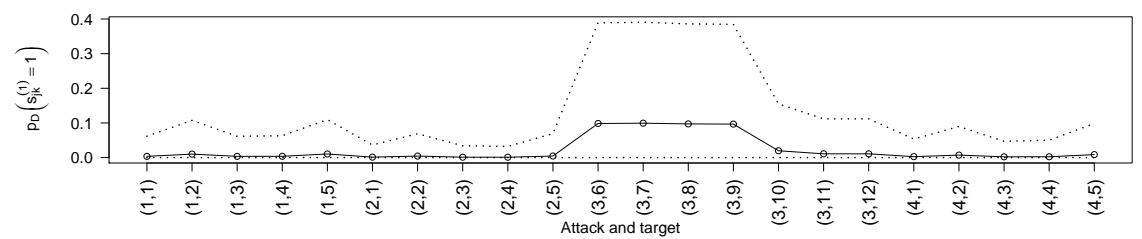

(a) Probability of successful attack.

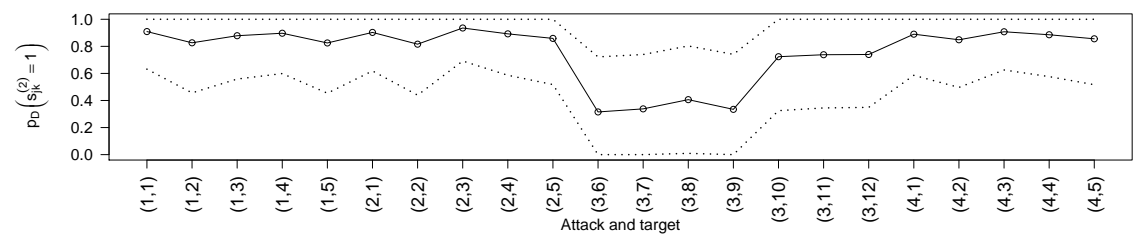

(b) Probability of successful recovery.

Figure 8: Analysing the Attacker's strategy. 
As for the chance of successful recovery, those targets protected with mobile resources have similar probabilities, around 0.7-0.9, regardless of the type of attack, see Figure 8b. On the contrary, when the attack is en route, the recovery action is less likely to be successful. This is reasonable, since attack $a_{3}$ is, in general, launched far from protected places, such as stations or hotspots. Therefore, mobile resources have to be shifted from the nearest point, diminishing their efficacy.

\subsection{Sensitivity analysis}

There are many potential sensitivity analysis issues that could be explored. We illustrate only studying whether hiring a second helicopter might be useful.

From the above results, it is clear that terrorists would most likely choose an attack $a_{3}$ against tracks, due to its higher success rate and the slightly lower chances of being killed or captured. Helicopters would focus on protecting the tracks, but their high cost penalises the Defender's expected utility. Indeed, including a second unit would imply drastic reductions in other resources, due to the budget constraint: a helicopter is approximately as costly as hiring 37 guards or installing 14 detectors. We explore now whether a more effective protection provided by helicopters would make the lease of a second unit worthwhile.

We rerun our simulations changing $\gamma_{7}$ from 1.54 (Table 6) to 3 , which-according to experts - could be achieved through additional specialised crew training and redefining patrolling routes, both actions entailing relatively small costs. Under this reconfigured setting, the optimal portfolio is similar to the earlier one, still with one helicopter, and simply reallocating resources to different locations. However, the expected utility is worse in this case, due to the following chain of facts. First, terrorists would find attack $a_{3}$ less attractive - due to the greater effectiveness of helicopters - although it would still be their most likely choice. There would be, in turn, higher chances that the Attacker would prefer alternative attacks, which would expectedly cause heavier damage to the Defender. Yet those portfolios including a second helicopter would have worse expected utilities than the optimal one. This can be explained through a similar reasoning. More than half of the budget would be now spent in helicopters, which are only useful to protect tracks against attack $a_{3}$ : all other targets would be considerably more vulnerable to attacks $a_{1}, a_{2}, a_{4}$, 
entailing more severe consequences to the Defender.

\section{Discussion}

We have analysed the protection of CNIs from terrorist attacks. We have considered a generic network in which value can be found at nodes, links and hotspots, the latter regarded as locations within the links particularly important for the Defender and/or the Attacker. We have deployed an ARA model over each relevant target in the network, relating the models through resource constraints and aggregation of results over various sites. A Sequential Defend-Attack-Defend model has been used, although different models could be accommodated for different targets.

We have illustrated the methodology with a case study dealing with a section of the Spanish railway system. Through it, we have shown how ARA can support the railway operator in choosing her best protection strategy against terrorist attacks trying to: (i) Deter terrorists; (ii) Minimise their chances of succeeding; and (iii) In case of a successful attack, consider the recovery decision to capture the terrorists. The case study may be used as a template for other CNI protection problems.

The model chosen is dynamic, in the sense that we have allowed for mobility of resources for the Defender after a successful attack. It could be further sophisticated at an operational level, by deciding appropriate patrolling schedules for the mobile resources, with models as in [38], 39], or [40], once we have decided with our model the best resource allocation.

Note that we have explicitly disregarded possible cascading effects resulting from terrorist actions, in the sense that we assume that the impact over one target will not propagate along the network. This could be relevant for e.g. communication or energy networks, see 41] or 42] for related applications. In our case, attackers could perform only a single attack, but these could be more resourceful and launch several attacks, as in the Madrid bombings. This complicates the problem combinatiorally affecting the simulations required to forecast attacks. Additional problems appear when there are multiple attackers. Other relevant modelling variants have been mentioned in the main text.

Finally, a computational environment supporting the implementation of ARA models 
would be useful for practitioners.

\section{Acknowledgements}

Work supported by the Spanish Ministry of Economy and Innovation program MTM2014-56949-C3-1-R, the ESF-COST Action IS1304 on Expert Judgement and the AXAICMAT Chair on Adversarial Risk Analysis. It also received funding from the European Union's Seventh Framework Programme for Research, Technological Development and

Demonstration under grant agreement no 285223. Discussions with referees are gratefully acknowledged.

\section{References}

[1] World Economic Forum, World Economic Forum. Global Risks (2015). URL http://www3.weforum.org/docs/WEF_Global_Risks_2015_Report15.pdf

[2] B. C. Ezell, S. P. Bennett, D. von Winterfeldt, J. Sokolowski, A. J. Collins, Probabilistic risk analysis and terrorism risk, Risk Anal 30 (4) (2010) 575-589.

[3] L. M. Wein, OR Forum - Homeland Security: From Mathematical Models to Policy Implementation, Oper Res 57 (4) (2009) 801-811.

[4] T. G. Lewis, Critical Infrastructure Protection in Homeland Security: Defending a Networked Nation, John Wiley \& Sons, New Jersey, 2006.

[5] G. Brown, M. Carlyle, J. Salmerón, K. Wood, Defending critical infrastructure, Interfaces 36 (6) (2006) 530-544.

[6] Y. Haimes, T. Longstaff, The role of risk analysis in the protection of critical infrastructures against terrorism, Risk Anal 22 (3) (2002) 439-444.

[7] W. Enders, T. Sandler, The Political Economy of Terrorism, 2nd edition, Cambridge University Press, New York, 2011.

[8] G. S. Parnell, D. Banks, L. Borio, G. Brown, L. A. T. Cox Jr, J. Gannon, E. Harvill, H. Kunreuther, S. Morse, M. Pappaioanou, S. Pollock, N. Singpurwalla, A. Wilson, 
Report on Methodological Improvements to the Department of Homeland Security's Biological Agent Risk Analysis, National Academies Press (2008).

[9] K. Hausken, J. Zhuang, Governments' and terrorists' defense and attack in a Tperiod game, Decis Anal 8 (1) (2011) 46-70.

[10] X. Shan, J. Zhuang, Subsidizing to disrupt a terrorism supply chain - a four-player game, J Oper Res Soc 65 (7) (2014) 1108-1119.

${ }_{545}$ [11] S. P. Hargreaves-Heap, Y. Varoufakis, Game Theory: A Critical Introduction, 2nd Edition, Routledge, London, 2004.

[12] D. Ríos Insua, J. Ríos, D. Banks, Adversarial risk analysis, J Am Stat Assoc 104 (486) (2009) 841-854.

[13] K. Hausken, G. Levitin, Review of systems defense and attack models, Int J Perform Eng 8 (4) (2012) 355-366.

[14] M. Dziubiński, S. Goyal, Network design and defence, Game Econ Behav 79 (2013) $30-43$.

[15] G. S. Parnell, C. M. Smith, F. I. Moxley, Intelligent adversary risk analysis: A bioterrorism risk management model, Risk Anal 30 (1) (2010) 32-48.

555 [16] J. Ríos, D. Ríos Insua, Adversarial risk analysis for counterterrorism modeling, Risk Anal 32 (5) (2012) 894-915.

[17] M. R. Haberfeld, A. von Hassell, A New Understanding of Terrorism: Case Studies, Trajectories and Lessons Learned, Humanities, Social Sciences and Law, Springer, New York, 2009.

[18] BBC News Europe, France train attack: Europe 'to tighten rail security', Press Release, http://www.bbc.com/news/world-europe-34092544 (August 2015).

[19] R. D. Shachter, Evaluating influence diagrams, Oper Res 34 (6) (1986) 871-882.

[20] J. P. C. Kleijnen, R. G. Sargent, A methodology for fitting and validating metamodels in simulation, Eur J Oper Res 120 (1) (2000) 14-29. 
[21] S. French, D. Ríos Insua, Statistical Decision Theory, Arnold, London, 2000.

[22] BBC News Europe, Spain arrests al-Qaeda in Islamic Maghreb suspect, Press Release, http://www.bbc.com/news/world-europe-14563948 (August 2011).

[23] BBC News Europe, 'Al-Qaeda trio' arrested in southern Spanish towns, Press Release, http://www.bbc.com/news/world-europe-19091753 (August 2012).

[24] New York Post, Spain, Morocco arrest 9 in ISIS terror cell, Press Release, http://nypost.com/2014/09/26/spain-morocco-arrest-9-in-isis-terror-cell/ (September 2014).

[25] El País, Tracking the threat of Spain's returning jihadists, Press Release, http://elpais.com/elpais/2015/06/30/inenglish/1435665322_643214.html （July 2015).

[26] Daily Mail, Teenager paraded down the street in a full burqa and handcuffs after Spanish police arrested her for 'recruiting other women to fight for ISIS in Syria', Press Release, http://www.dailymail.co.uk/news/article-3223565/ (September 2015).

[27] G. L. Keeney, D. von Winterfeldt, Identifying and structuring the objectives of terrorists, Risk Anal 30 (12) (2010) 1803-1816.

[28] R. L. Keeney, Modeling values for anti-terrorism analysis, Risk Anal 27 (3) (2007) $585-596$.

[29] A. Riera Font, A. Ripoll Penalva, J. Mateu Sbert, Estimación del valor estadístico de la vida en España: Una aplicación del método de salarios hedónicos, Hacienda Pública Esp 2 (181) (2007) 29-48.

[30] M. Buesa Blanco, A. Valiño Castro, J. Heijs, T. Baumert, J. González Gómez, Evaluación del coste directo de los atentados terroristas del 11-M para la economía de la Comunidad de Madrid. Documento de trabajo no. 51, Tech. rep., Instituto de Análisis Industrial y Financiero, UCM (2005).

[31] J. S. Dyer, R. K. Sarin, Relative risk aversion, Manage Sci 28 (8) (1982) 875-886. 
[32] P. H. Farquhar, State of the art-utility assessment methods, Manage Sci 30 (11) (1984) 1283-1300.

[33] W. K. Viscusi, J. E. Aldy, The value of a statistical life: a critical review of market estimates throughout the world, J Risk Uncertain 27 (1) (2003) 5-76.

[34] W. K. Viscusi, Valuing risks of death from terrorism and natural disasters, J Risk Uncertain 38 (3) (2009) 191-213.

[35] P. Müller, D. Ríos Insua, Issues in Bayesian analysis of neural network models, Neural Comput 10 (3) (1998) 749-770.

[36] G. Cybenko, Approximation by superpositions of a sigmoidal function, Mathematics of Control, Signals and Systems 2 (4) (1989) 303-314.

[37] D. E. Goldberg, Genetic Algorithms in Search, Optimization, and Machine Learning, Addison-Wesley, Reading, MA, 1989.

[38] S. Alpern, A. Morton, K. Papadaki, Patrolling games, Oper Res 59 (5) (2011) 12461257.

[39] M. Brown, S. Saisubramanian, P. R. Varakantham, M. Tambe, STREETS: Gametheoretic traffic patrolling with exploration and exploitation, in: Innovative Applications in Artificial Intelligence (IAAI), Twenty Eighth AAAI Conference on Artificial Intelligence, AAAI-14, Research Collection School Of Information Systems, 2014.

[40] N. Zoroa, M. J. Fernández-Sáez, P. Zoroa, Patrolling a perimeter, Eur J Oper Res 222 (3) (2012) 571-582.

[41] J. Salmerón, K. Wood, R. Baldick, Analysis of electric grid security under terrorist threat, IEEE T Power Syst 19 (2) (2004) 905-912.

[42] Å. J. Holmgren, Using graph models to analyze the vulnerability of electric power networks, Risk Anal 26 (4) (2006) 955-969. 